\title{
The demands of cancer survivorship: the who, what, when, where, why, and how
}

\author{
Jennifer R. Klemp, PhD, MPH, Catherine Knight, Lori Ranallo, RN, MSN, ARNP, \\ and Carol J. Fabian, MD \\ University of Kansas Cancer Center, Breast Cancer Survivorship Center, Kansas City
}

$\mathrm{W}$ ith an exponential increase in the number of cancer survivors over the past few decades, we have an opportunity and responsibility to effectively manage cancer survivors across the continuum of cancer care. The delivery of survivorship care requires realistic deliverables with defined outcomes that focus on cost, impact on disease management and prevention, and integration within a health care delivery model. Building a framework using defined time-points and definitions can be helpful. Due to the complex nature of delivering cancer survivorship care, it is necessary to establish collaborations with specialty providers including cardiologists, reproductive specialists, endocrinology, ophthalmology, allied health professionals and cancer rehab, to name a few. Strengthening relationships with primary care providers will enhance the transition from cancer care to primary care. Essential tools to help fulfill these goals and achieve national standards include using expert recommended treatment summaries and survivorship care plans. These tools support a shared care model with the goal of high quality, coordinated healthcare for the survivorship population. With limited evidence to guide the delivery of survivorship care and national standards looming, how do we meet the demands of cancer survivorship? This article explores the "the who, what, when, where, why and how?" of cancer survivorship care.

\section{Who are cancer survivors?}

According to the National Coalition for Cancer Survivorship, a cancer survivor "is anyone who has been diagnosed with cancer - from the time of

Correspondence Jennifer R. Klemp, PhD, MPH, University of Kansas Cancer Center, Division of Clinical Oncology, 3901 Rainbow Boulevard Kansas City, KS 66160 (jklemp@kumc. edu).

Disclosures Dr. Klemp has no financial disclosures. She is the founder and CEO of Cancer Survivorship Training, an e-learning solutions company. diagnosis and for the balance of his or her life." This definition helps to provide an identity to those diagnosed with cancer, however, many patients with advanced disease may not identify as a cancer survivor. At present, there are close to 14 million cancer survivors with a projected 18 million by 2022 . The 5 -year relative survival rate for all cancers diagnosed between 2002 and 2008 is $68 \%$, up from $49 \%$ in $1975-1977$, primarily due to improvements in early detection and more effective therapeutic options. ${ }^{2}$

\section{Who delivers care to cancer survivors?}

Survivorship care is by nature multidisciplinary and ideally provided using a shared-care approach. ${ }^{3}$ The central locus of control is the survivor, who must be empowered with information and resources to employ self-management strategies and play an active role in their health care. The cancer care team may include an oncologist, surgeon, radiation oncology, midlevel providers, nurses, allied health professionals, supportive care services, and caregivers, along with other specialty and primary care providers. This complex system requires coordination and communication among providers and with the survivor. During the acute phase of treatment, the cancer care team assumes more responsibility but over time, this transitions back to primary care. Initiatives to incorporate elements of cancer survivorship into postgraduate training and continuing medical education are essential to develop a workforce equipped to manage the ongoing needs of cancer survivors. ${ }^{4-6}$

\section{What is survivorship care?}

Survivorship care often refers to the posttreatment phase of the cancer care trajectory. Survivorship care is dynamic and aims to meet each survivors' (c) 2013 Frontline Medical Communications 


\begin{tabular}{|c|c|}
\hline \multicolumn{2}{|c|}{ Physical } \\
\hline Long-term & Late \\
\hline Fatigue & $\begin{array}{l}\text { Second primary } \\
\text { cancer }\end{array}$ \\
\hline Endocrine issues & $\begin{array}{l}\text { Cardiac or pulmonary } \\
\text { dysfunction }\end{array}$ \\
\hline Infertility & $\begin{array}{l}\text { Osteoporosis/endocrine } \\
\text { issues }\end{array}$ \\
\hline Cognitive impairment & Cognitive impairment \\
\hline Neuropathy & Cataracts \\
\hline $\begin{array}{l}\text { Change in weight (loss } \\
\text { or gain) }\end{array}$ & Weight gain \\
\hline Organ problems & Lymphedema \\
\hline Chronic pain & Oral health issues \\
\hline Sexual health issues & Sexual health issues \\
\hline \multicolumn{2}{|c|}{ Psychosocial and existential, long-term and late } \\
\hline \multicolumn{2}{|c|}{$\begin{array}{l}\text { Psychological, depression, anxiety (fear of recurrence), } \\
\text { isolation, altered body image }\end{array}$} \\
\hline \multicolumn{2}{|c|}{$\begin{array}{l}\text { Social, changes in interpersonal relationships, concerns } \\
\text { about health insurance, job change/loss, return to } \\
\text { work/school, financial burden }\end{array}$} \\
\hline $\begin{array}{l}\text { Existential, sense of purp } \\
\text { hope, appreciation of }\end{array}$ & $\begin{array}{l}\text { ning, role of religion, } \\
\text { inty }\end{array}$ \\
\hline
\end{tabular}

unique physical, social, psychosocial and spiritual needs. Meeting those needs includes identifying and managing long-term and late side effects of treatment. Long-term side effects of treatment (chemotherapy, surgery, radiation therapy) include those identified during diagnosis and treatment, and may persist post-acute treatment. ${ }^{7}$ Most long-term side effects lessen over time. Late side effects are identified once acute treatment is completed and might occur a few months or years post treatment. Examples of physical long-term and late effects of cancer and psychological, social, and existential long-term and late effects are listed in Table 1.

\section{When is survivorship care delivered?}

Survivorship care generally refers to a distinct phase in the cancer care trajectory and into long-term posttreatment care (Figure 1). ${ }^{8}$ The model described by the Institute of Medicine (IOM) presents survivorship care during the disease managed or disease free interval of the continuum of cancer care. ${ }^{3}$ However, the trajectory has been modified to describe how delivering education and preventive strategies during the acute treatment phase may provide early intervention and mitigation. In addition, the figure highlights an opportunity to address the unmet needs of survivors with advanced disease under the umbrella of survivorship care.

Building a framework using defined time-points and definitions, and utilization of resources and costs can be helpful. For example, standardizing follow-up visits within 3 months of ending treatment to include a treatment summary and initial survivorship care planning can help meet American Society of Clinical Oncology's (ASCO's) Quality Oncology Practice Initiative (QOPI) standards and can facilitate the management of survivorship-related issues and enhance communication. ${ }^{9}$

\section{Where is survivorship care delivered?}

There have been numerous articles written highlighting delivery models of care and the need to standardize the delivery of care. Very little data to date highlights outcomes of the delivery of survivorship care and what is really looks like in practice. Table 2 provides a comprehensive overview of survivorship care models of delivery. The challenge with choosing a single delivery model may be complicated by the individual needs of the survivor. If we are providing personalized survivorship care, organizations may try and align with a single model of delivery, but maintain the flexibility to meet the individual survivors' needs. Therefore, in order to assess the impact of survivorship care, focusing on defined outcomes (including patient centered outcomes and costs) might make more sense than on the model of delivery itself.

Also, conceptualizing cancer survivorship using the chronic health care model (similar to the management of cardiovascular disease and diabetes), ${ }^{10}$ can provide necessary framework for the delivery of survivorship care. This may also include incorporation of the oncology patient centered medical home ${ }^{11}$ and other models of delivery that focus on patient centered outcomes and coordinated care.

\section{Why do we need to deliver survivorship care?}

The impact of cancer has been the focus of research in both pediatric and adult populations. With a critical mass of cancer survivors and evidence to support the ongoing needs of cancer survivors, expert consensus recommendations have been developed to guide the delivery of care to cancer survivors. In 2005, the IOM highlighted that survivorship care is a neglected phase of the cancer care trajectory. Also, providers lack education and training to manage cancer survivors. Additional barriers to care include fragmentation and limited evidence-based guidelines to direct follow-up care (Figure 2).

Recent clinical practice guidelines developed by evidence based expert consensus were released by the National Comprehensive Cancer Network (NCCN) on 


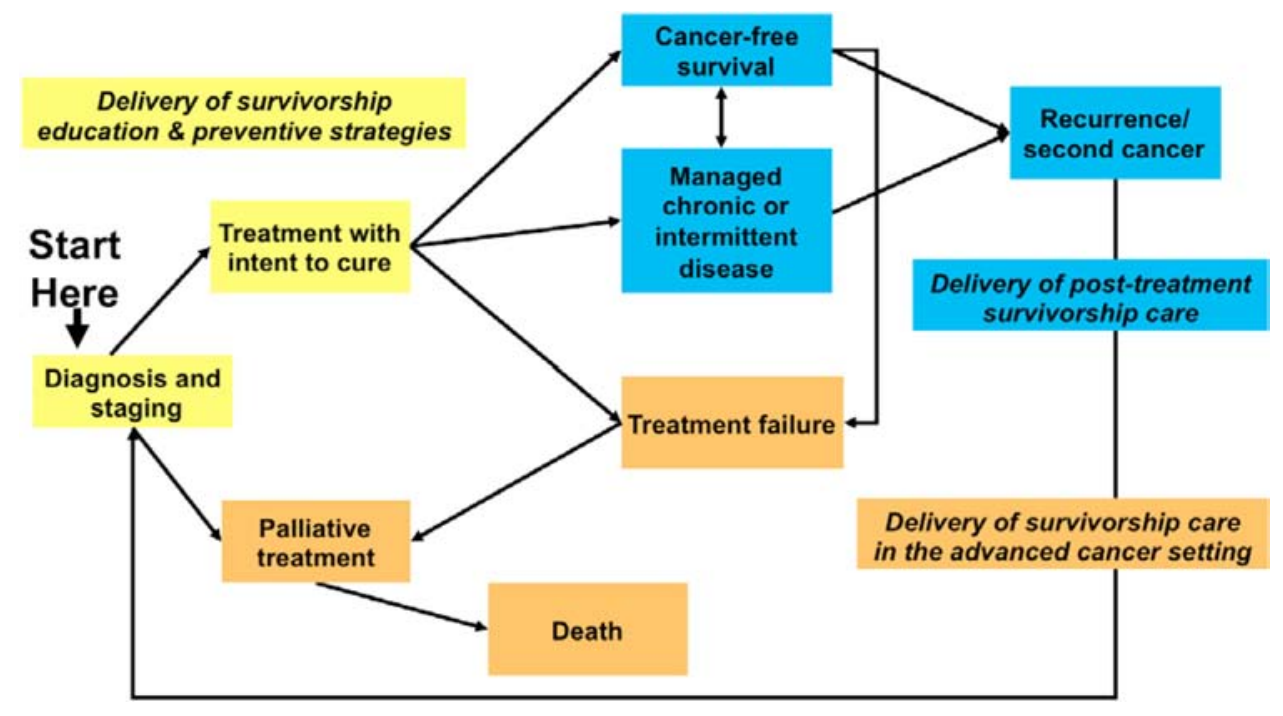

FIGURE 1 Cancer care trajectory. Adapted from the Institute of Medicine. From Cancer Patient to Cancer Survivor: Lost in Translation. http://www.nap.edu/catalog.php?record_id=11468. Accessed July 25, $2011^{19}$ one of the models described in $\mathrm{Ta}-$ ble 2. However, as many who practice survivorship care know, one size does not fit all. It is common to use a central framework for workflow but maintain flexibility to meet the individual needs of survivors based on comorbidities, risk of recurrence, access to resources, geographic area, just to name a few.

For example, at the University of Kansas Cancer Center, ${ }^{14}$ the delivery of survivorship care is integrated within the current clinical setting. This includes disease specific survivorship clinics within the academic medical center and a general survivorship clinic within the community practice setting. The delivery of treatment summaries and care plans, and continuity of care, is primarily delivered by midlevel pro-

Survivorship (Version 1/2013). ${ }^{12}$ These guidelines cover topics including assessment for anxiety and depression, cognitive function, exercise, fatigue, immunizations and infections, pain, sexual function, and sleep disorders. These guidelines are designed to provide a framework for general survivorship care and management and are not intended to provide specific guidelines on the surveillance and follow-up requirements for a survivor's primary care. In addition, the Children's Oncology Group and ASCO both provide evidence consensus recommendations for clinical practice guidelines for certain types of cancer. These practice guidelines, along with cancer screening guidelines and health promotion from organizations including the American Cancer Society, serve as part of a comprehensive framework in the delivery of survivorship care. Having access to guidelines and recommendations promotes the delivery of patient centered, coordinated care, and requires ongoing evaluation of outcomes.

\section{How is survivorship care delivered?}

Organizations including $\mathrm{ASCO}^{9}$ and the American College of Surgeons Commission on Cancer Program Standards for 2012 (implementation by 2015) ${ }^{13}$ have encouraged the use of treatment summaries and care plans to address the gap of communication and coordination of care. These tools should be integrated into the electronic health record and provide a convenient way to store information about the type and stage of cancer, cancer treatment, and follow-up care. In addition, these tools require a health care delivery system to make the plan become a reality. Survivorship care is often delivered using viders. Transition to the survivorship continuity clinic is based on risk stratification, with those at lowest risk of recurrence and late effects transitioning earlier than those at highest risk. There is a focus on maintaining a relationship with primary care, which is vital for shared care delivery or ultimate transition to primary care.

Continuing medical education and training is facilitated using multiple tools. To provide instruction among mid-level providers, a train-the-trainer model is used to share workflow and documentation within the EHR, delivery of care, access to resources, use of navigation, and so on. In addition, we have built an e-learning platform, www.cancersurvivorshiptraining.com, ${ }^{5}$ which provides cancer care providers and collaborating health care professionals with access to expert developed continuing medical education on topics of cancer survivorship, regardless of geographic or practice location.

Lastly, it is important to develop referrals to specialists, including but not limited to cardio-oncology, sexual medicine, cancer rehabilitation, support services, and community resources. This shared care delivery will provide the necessary health care delivery model to manage the ongoing needs of cancer survivors.

\section{Summary}

Incorporating survivorship care into practice is not an easy undertaking, but a necessary one. With the growing demands of cancer survivorship, we are provided with an opportunity to develop an infrastructure to manage cancer survivors across the continuum of cancer 


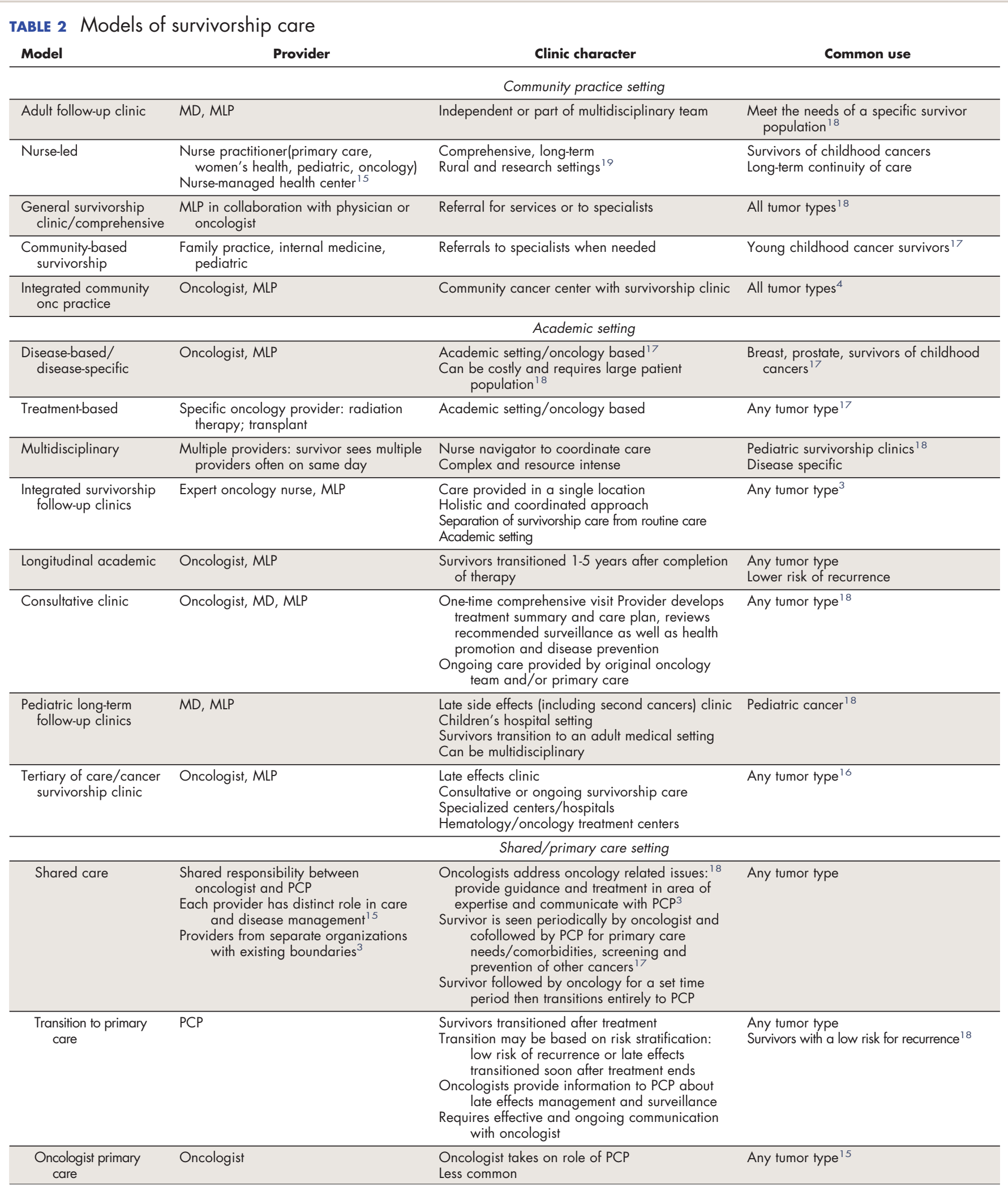

Abbreviations: MLP, midlevel provider; PCP, primary care provider. 


\section{Features}

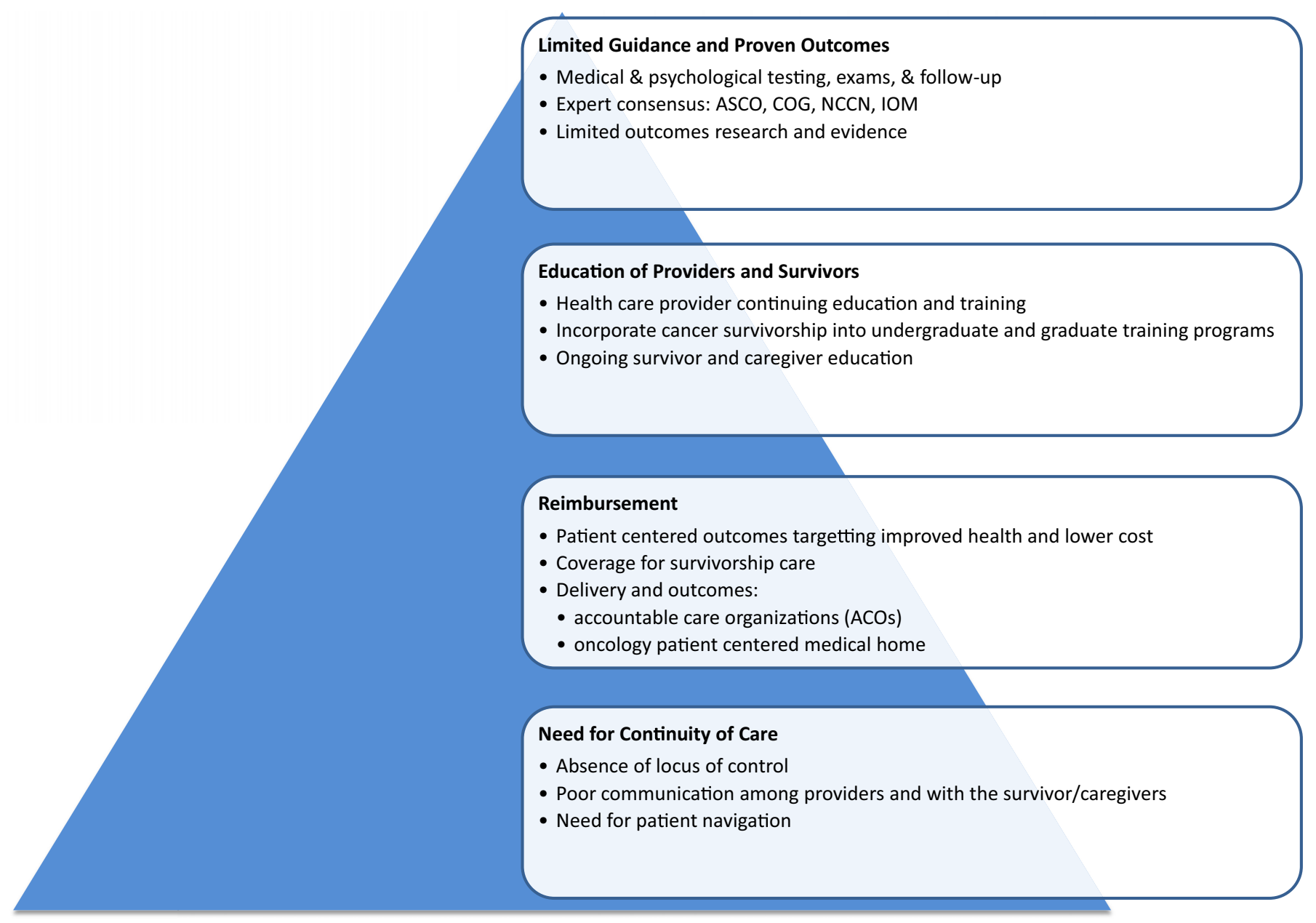

FIGURE 2 Barriers to delivering cancer survivorship care..$^{3,4,19}$

care. This includes developing definitions, implementing workflow, working through models of delivery and collaboration among providers, EHR developed treatment summaries and care plans, shared care with specialists and primary care, and ongoing evaluation. With limited evidence to support these efforts, not only is collaboration and evaluation essential, so is our need to depend on technology to develop workflows, aggregate treatment history, build survivorship care plans, communicate between providers, and satisfy meaningful use.

\section{References}

1. National Coalition for Cancer Survivorship. Our Mission. http://www. canceradvocacy.org/about-us/our-mission/. Accessed March 4, 2013.

2. American Cancer Society. Facts and Figures 2013. http://www.cancer. $\mathrm{org} /$ research/cancerfactsfigures/cancerfactsfigures/cancer-facts-figures2013. Accessed March 4, 2013.

3. Hewitt M, Greenfield S, Stovall E. From Cancer Patient to Cancer Survivor: Lost in Transition. Washington, DC: The National Academies Press; 2005.
4. McCabe M, Bhatia S, Oeffinger K, Reaman G, Tyne C, Wollins D, Hudson M. American Society of Clinical Oncology Statement: achieving high-quality cancer survivorship care. J Clin Oncol. 2013;31(5):631-640. doi: http://dx.doi.org/10.1200/JCO.2012. 46.6854.

5. Cancer Survivorship Training. http://www.cancersurvivorshiptraining. com/. Accessed March 4, 2013.

6. Klemp J, Frazier LM, Glennon C, Trunececk J, Irwin M. Improving cancer survivorship care: oncology nurses' educational needs and preferred methods of learning. J Cancer Educ. 2011;26(2): 234-242.

7. Aziz NM, Rowland JH. Trends and advances in cancer survivorship research: challenge and opportunity. Sem Radiat Oncol. 2003; 13(3):248-266.

8. Centers for Disease Control. The National Action Plan for Cancer Survivorship. http://www.cdc.gov/cancer/survivorship/what_cdc_is_ doing/action_plan.htm. Accessed March 4, 2013.

9. American Society of Clinical Oncology. Quality Oncology Practice Initiative (QOPI). http://qopi.asco.org/. Assessed March 4, 2013.

10. Improving Chronic Illness Care. The Chronic Care Model. http:// www.improvingchroniccare.org/index.pahp? $\mathrm{p}=$ The_Chronic_ Care_Model\&s=2. Accessed on April 21, 2013.

11. Sprandio JD. Oncology patient-centered medical home. J Oncol Pract. 2012;8(3S):47s-49s. 


\section{Survivorship}

12. National Comprehensive Cancer Network (NCCN) on Survivorship (Version 1/2013). www.nccn.org. Accessed April 21, 2013.

13. American College of Surgeons. Cancer Program Standards 2012, Version 1.1: Ensuring Patient-Centered Care. http://facs.org/cancer/ coc/programstandards2012.html. Accessed April 21, 2013.

14. The University of Kansas Cancer Center. http://www.kucancercenter. org. Accessed April 21, 2013.

15. Cooper J, Loeb S, Smith C. The primary care nurse practitioner and cancer survivorship care. J Am Acad Nurse Pract. 2010;22(8):394-402. doi: http://dx.doi.org/10.1111/j.17457599.2010.00528.x.
16. Ganz P, Hahn E. Implementing a survivorship care plan for patients with breast cancer. I Clin Oncol. 2008;26(5):759-767. doi: 10.1200/jco.2007.14.2851.

17. Landier W. Survivorship care: essential components and models of delivery. Oncology. 2009;23(4):46-53. http://search.proquest.com/ docview/304677142? accountid=28920. Accessed March 14, 2013.

18. McCabe MS, Jacobs LA. Clinical update: survivorship care - models and programs. Semin Oncol Nurs. 2012;28(3):e1-8. doi: http:// dx.doi.org/10.1016/j.soncn.2012.05.001.

19. Hewitt M, Ganz P. Implementing Cancer Survivorship Care Planning. Washington DC: The National Academies Press; 2007. 